\title{
Oxidised proteins and superoxide anion production in the diaphragm of severe COPD patients
}

\author{
J. Marin-Corral*,\#, J. Minguella*,\#, A.L. Ramírez-Sarmiento*, S.N.A. Hussain \\ J. Gea ${ }^{*,+,}$ and E. Barreiro ${ }^{*,+, \S}$
}

ABSTRACT: In the diaphragms of chronic obstructive pulmonary disease (COPD) patients, the nature of oxidatively modified proteins and superoxide anion production were explored.

Diaphragm specimens were obtained through thoracotomy because of localised lung lesions in COPD patients (16 severe and eight moderate) and 10 control subjects. Lung and respiratory muscle functions were evaluated. Oxidised proteins were identified using immunoblotting and mass spectrometry. Protein and activity levels of the identified proteins were determined using immunoblotting and activity assays. Lucigenin-derived chemiluminescence signals in a luminometer were used to determine superoxide anion levels in muscle compartments (mitochondria, membrane and cytosol) using selective inhibitors.

In severe COPD patients compared with controls, respiratory muscle function was impaired; creatine kinase, carbonic anhydrase III, actin and myosin were oxidised; myosin carbonylation levels were increased five-fold; creatine kinase content and activity and myosin protein were reduced; superoxide anion levels were increased in both mitochondria and membrane compartments; and the percentage of superoxide anion inhibition achieved by rotenone was significantly greater.

In severe COPD patients, oxidation of diaphragm proteins involved in energy production and contractile performance is likely to partially contribute to the documented respiratory muscle dysfunction. Furthermore, generation of the superoxide anion was increased in the diaphragms of these patients.

KEYWORDS: Chronic obstructive pulmonary disease, muscle compartments, oxidised proteins, respiratory muscles, superoxide anion

hronic obstructive pulmonary disease (COPD) is a highly prevalent condition that imposes a substantial burden in terms of disability and impairment of quality of life [1]. COPD is also a leading cause of death worldwide and is projected to increase within the next decade, since the prevalence of cigarette smoking continues to rise and the population ages $[2,3]$. Poor exercise tolerance and reduced quality of life, partially owing to muscle dysfunction, are major complaints in patients with COPD.

Several reports have shown that in severe COPD the main inspiratory muscle, the diaphragm, undergoes adaptive modifications that probably render the overloaded muscle more resistant to fatigue [4-8]. However, there is growing evidence that in vitro fibre contractile function is also impaired in the diaphragms of patients with COPD [9], even at early stages of their disease, as shown by reduced myosin heavy chain (MyHC) content and increased protein degradation via the ubiquitin-proteasome pathway [10, 11]. Oxidative stress, among other factors, was also suggested to be a contributor to this process of muscle dysfunction and wasting [12-14], although its specific role is yet to be fully elucidated. The oxidative post-translational modifications of proteins may cause loss of protein function as a result of oxidation of residues in the protein active site. In other cases, oxidation of critical residues may lead to increased proteolytic degradation of the modified proteins [15]. Indeed, it has also been suggested that the
AFFILIATIONS

${ }^{*}$ Muscle Research and Respiratory System Unit, Respiratory Medicine and Thoracic Surgery Depts, IMIMHospital del Mar, Barcelona,

${ }^{\text {\#S} S c h o o l ~ o f ~ M e d i c i n e, ~ U n i v e r s i t a t ~}$ Autònoma de Barcelona,

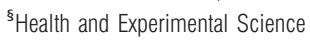
Department (CEXS), Universitat

Pompeu Fabra, Barcelona, and ${ }^{+}$Centro de Investigación en Red de Enfermedades Respiratorias (CIBERES), Spain.

"Critical Care and Respiratory Divisions, Royal Victoria Hospital and Meakins-Christie Laboratories, McGill University, Montreal, QC, Canada.

CORRESPONDENCE

E. Barreiro, Muscle and Respiratory System Research Unit, IMIM-

Hospital del Mar, PRBB, Dr. Aiguader 88, E-08003 Barcelona, Spain. Fax: 34933160410

E-mail: ebarreiro@imim.es

Received:

May 102008

Accepted after revision:

January 172009

\section{SUPPORT STATEMENT}

This study was supported by FIS, the Spanish Health Ministry (grants 04/ 00161 and 06/1043), SEPAR 2004, the Catalan Government (2005SGR01060) and CIBERES (all Spain) E. Barreiro received the ERS COPD Research Award 2008.

STATEMENT OF INTEREST None declared. 
reactive oxygen species (ROS) cascade regulates pathophysiological signalling, leading to proteolysis and apoptosis when synthesised at high levels within the myofibres [16].

In a previous study [14], it was shown that diaphragm oxidative stress could be a contributor to the intrinsic respiratory muscle dysfunction of severe COPD patients. However, in that study, neither the nature of the oxidatively modified proteins nor the generation of ROS within the diaphragm fibres of COPD patients were explored. On this basis, it is now hypothesised that excessive ROS production in the diaphragm muscles of severe COPD patients chronically exposed to inspiratory overloads would target specific proteins involved in muscle contractile function, thereby contributing to the recognised in vivo ventilatory muscle dysfunction. Accordingly, our objectives were: first, to identify the nature of the oxidatively modified proteins (protein carbonylation) in the diaphragms of patients with both severe and moderate COPD and in control subjects; and, secondly, to explore the generation of superoxide anion within several muscle compartments in the diaphragms of the same patients and control individuals.

\section{METHODS}

For additional information concerning the Methods section, please refer to the online supplementary material.

\section{Subjects}

In total, 24 Caucasian male patients with stable COPD (eight moderate and 16 severe patients) [17] and 10 healthy male agematched sedentary controls were recruited on an out-patient basis (see online supplementary material for additional information). Moderate and severe COPD were defined in accordance with the Global Initiative for Chronic Obstructive Lung Disease guidelines [17]. All subjects underwent thoracotomy for a localised lung neoplasm. All patients were clinically stable at the time of the study. Eight COPD patients (three moderate and five severe) also participated in a former study aimed at assessing the associations between oxidative stress and respiratory muscle dysfunction in COPD [14]. The current investigation was designed in accordance with both the ethical standards on human experimentation of the IMIMHospital del Mar (Barcelona, Spain) and the World Medical Association guidelines for research on human beings. The Ethics Committee on Human Investigation at IMIM-Hospital del Mar approved all experiments. Informed written consent was obtained from all individuals.

\section{Nutritional and functional assessment}

Nutritional evaluation included body mass index (BMI) and analytical parameters. Both pulmonary and respiratory muscle functions were evaluated.

TABLE 1 Anthropometric characteristics and functional status of the study groups

\section{Control subjects}

Subjects $n$

Age yrs

BMI $\mathbf{k g} \cdot \mathrm{m}^{-2}$

FEV $1 \%$ pred

FVC \% pred

FEV $1 /$ FVC ratio

RV \%

TLC \% pred

RV/TLC ratio

FRC $\%$ pred

$D$ L,CO \% pred

KcO \% pred

$\mathrm{Pa}, \mathrm{O}_{2} \mathrm{KPa}$

$\mathrm{Pa}, \mathrm{CO}_{2} \mathrm{kPa}$

$P$ I,max \% pred

$P$ es, max $\mathrm{CmH}_{2} \mathrm{O}$

$P$ di, max $\mathrm{cmH}_{2} \mathrm{O}$

Type I fibres \%

Type II fibres \%

Hybrid fibres \%

Cross-sectional area $\mu \mathrm{m}^{2}$

Type I fibres

Type II fibres
Patients with moderate COPD

Patients with severe COPD

Data are presented as mean \pm SD, unless otherwise stated. BMI: body mass index; FEV1: forced expiratory volume in 1 s; \% pred: \% predicted; FVC: forced vital capacity RV: residual volume; TLC: total lung capacity; FRC: functional residual capacity; DL,Co: diffusing capacity of the lung for carbon monoxide; Kco: transfer coefficient of the lung for carbon monoxide; $\mathrm{Pa}, \mathrm{O}_{2}$ : arterial oxygen tension; $\mathrm{Pa}_{\mathrm{a}} \mathrm{CO}_{2}$ : arterial carbon dioxide tension; $\mathrm{Pl}$,max: maximal inspiratory pressure; $P$ es,max: maximal oesophageal pressure; Pdi,max: maximal transdiaphragmatic pressure. ${ }^{*}: p \leqslant 0.05, * *: p \leqslant 0.01 ; * \star *: p \leqslant 0.001$ between severe or moderate chronic obstructive pulmonary disease (COPD) patients and controls; ${ }^{*}: p \leqslant 0.05 ;{ }^{\# \#}: p \leqslant 0.01 ;{ }^{\# \# \#}: p \leqslant 0.001$ between severe and moderate COPD patients. 
a)

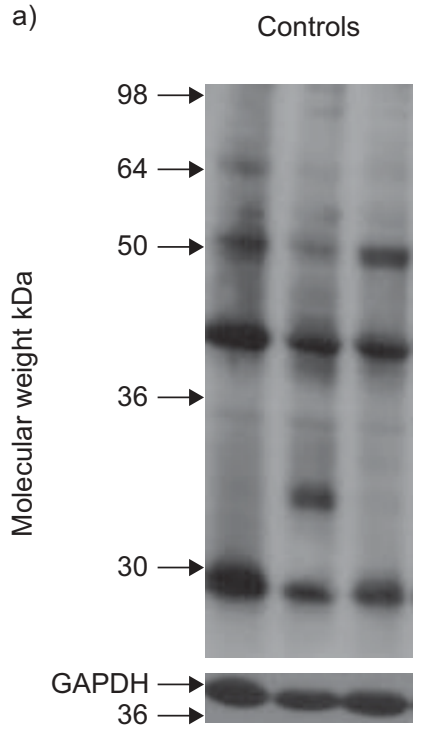

c)

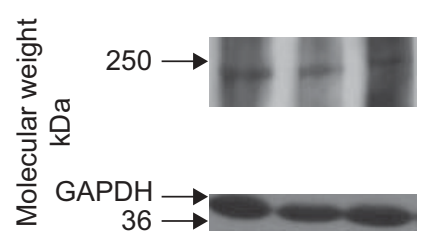

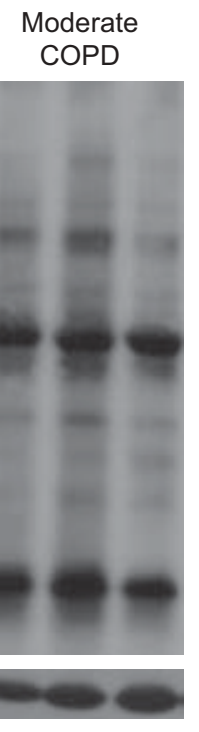

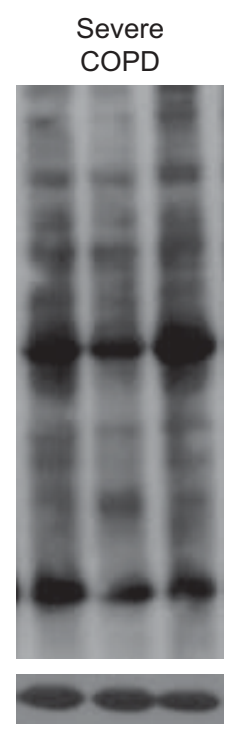

Moderate
COPD
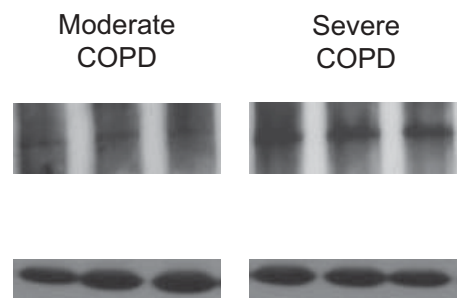
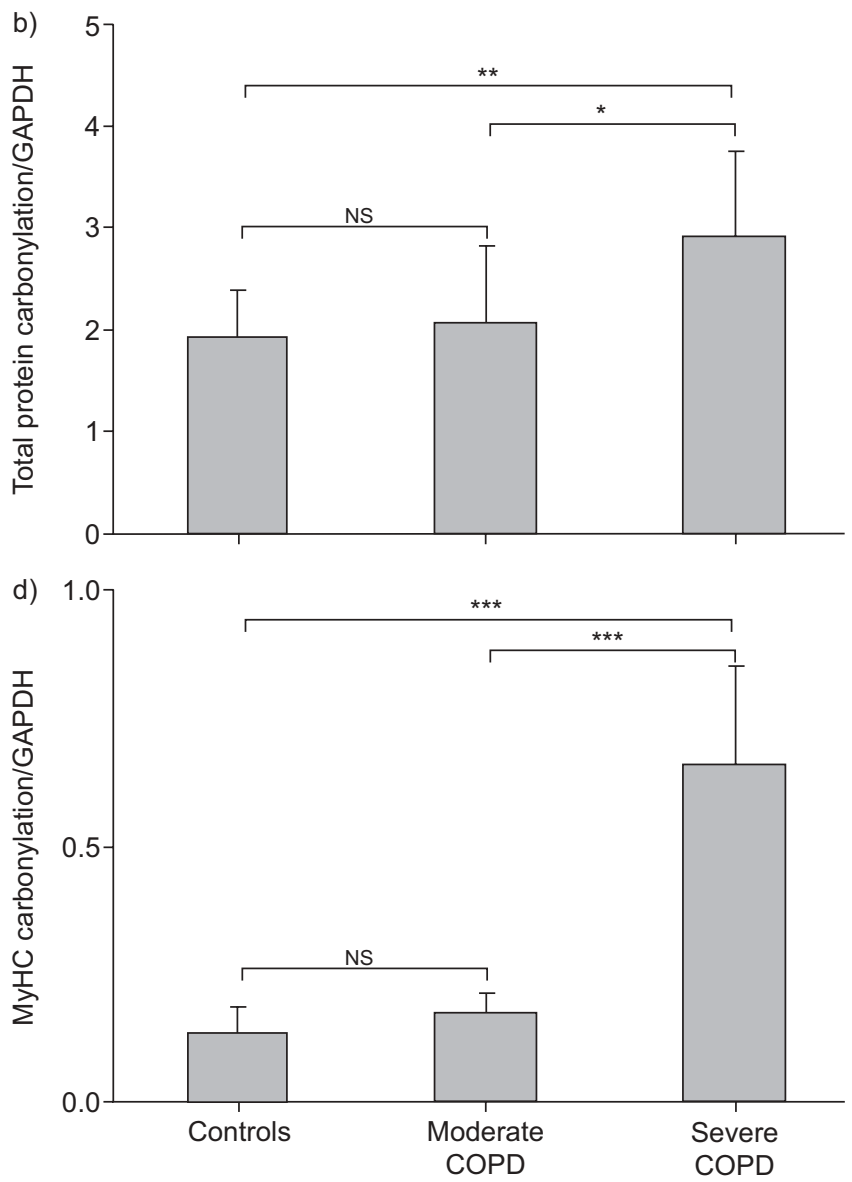

FIGURE 1. a) Representative examples of protein oxidation (total carbonyl groups) in diaphragms of control subjects $(n=10)$ and both moderate $(n=8)$ and severe $(n=16)$ chronic obstructive pulmonary disease (COPD) patients. Several protein carbonylated bands were detected. Monoclonal anti-GAPDH horseradish peroxidase (HRP)conjugated antibody was used to control equal loading among various lanes. b) Mean \pm SD values of total carbonyl formation were higher in the severe COPD patients ( $\mathrm{n}=16$ ) compared with either control subjects $(n=10)$ or moderate COPD patients. No significant differences were found in diaphragm protein carbonylation levels between moderate COPD patients and controls. c) Representative examples of carbonylated myosin heavy chain $(M y H C)$ protein in diaphragms of control subjects $(n=9)$ and both moderate $(n=8)$ and severe COPD patients $(n=9)$. Monoclonal anti-GAPDH HRP-conjugated antibody was used to control equal loading among various lanes. $d)$ Levels of carbonylated $\mathrm{MyHC}$ were greater in the diaphragms of the severe COPD patients compared with either controls or moderate patients. Carbonylation levels of MyHC did not differ between moderate patients and the controls. *: $p \leqslant 0.05 ;{ }^{* *}: p \leqslant 0.01 ; * * *: p \leqslant 0.001$. NS: nonsignificant.

\section{Biopsies}

During thoracotomy, because of localised lung lesions, diaphragm biopsy specimens were obtained from the anterior costal diaphragm lateral to the insertion of the phrenic nerve $[5,14]$.

\section{Biological muscle studies}

Immunoblotting of one-dimensional electrophoresis

The levels of oxidative and nitrosative stress and those of muscle creatine kinase, carbonic anhydrase III, $\alpha$-actin and $\mathrm{MyHC}$ were assessed, as described elsewhere [13, 14, 18].

Identification of carbonylated proteins: one-dimensional and twodimensional electrophoresis and mass spectrometry

Oxidised proteins (protein carbonylation) were separated and identified in the diaphragms of COPD patients and control subjects, as previously described [11, 13, 18].

Creatine kinase activity assay

Total muscle creatine kinase activity was measured with a commercial kit (Diagnostic Chemicals Ltd, Charlotte Town, PE, Canada), as described elsewhere [13, 18].
Muscle fibre counts and morphometry

Proportions of type I, type II and hybrid fibres were determined, as previously described [14]. The size of type I and type II fibres was also measured.

Measurements of superoxide anion production by lucigeninderived chemiluminescence

Lucigenin-derived chemiluminescence signals were determined in all muscle samples using a luminometer as formerly described $[19,20]$.

\section{Statistical analysis}

Data are presented as mean \pm SD. One-way ANOVA together with Tukey's test to adjust for multiple comparisons was employed in order to compare variable results among the three groups. Pearson's correlation coefficient was employed to assess relationships among different variables within COPD patients. A p-value $\leqslant 0.05$ was considered significant. 


\begin{tabular}{|c|c|c|c|c|}
\hline Identified carbonylated proteins & Accession number & Mass & MASCOT score & Peptide matched \\
\hline Muscle creatine kinase & gi/21536288 & 43302 & 207 & 38 \\
\hline Carbonic anhydrase III & gi/224979 & 29707 & 182 & 50 \\
\hline$\alpha-1$ actin precursor & $\mathrm{gi} / 4501881$ & 42366 & 117 & 29 \\
\hline
\end{tabular}

\section{RESULTS}

\section{Characteristics of the study subjects}

Table 1 indicates the main characteristics of the study subjects. No significant differences in age or nutritional status, as assessed by BMI and analytical parameters, were observed between control subjects and either severe or moderate COPD patients. However, forced expiratory volume in $1 \mathrm{~s}$ (FEV1), forced vital capacity (FVC), the FEV1/FVC ratio, diffusion capacity and arterial oxygen tension were significantly lower, while residual volume (RV) and the RV/total lung capacity ratio were significantly higher in severe COPD patients. Global respiratory muscle strength, as measured by maximal inspiratory pressure $(P \mathrm{I}, \mathrm{max})$ and maximal transdiaphragmatic pressure (Pdi,max), was moderately reduced in severe COPD patients.

\section{Muscle structure}

In the diaphragms of severe COPD patients, proportions of both type I and hybrid fibres were higher, while those of type II fibres were lower than in the muscles of either moderate
COPD or control subjects (table 1). When considering all COPD patients as a group, proportions of type I fibres negatively correlated with FEV1 $(\mathrm{r}=-0.499, \mathrm{p}=0.042)$.

\section{Identification of carbonylated proteins}

Total carbonyl group formation

As illustrated in figure 1a, anti-dinitrophenyhydrazone antibody detected different positive protein bands, with apparent masses ranging from 67 to $29 \mathrm{kDa}$, in the muscles of both patients and controls. The diaphragms of severe COPD patients showed higher levels of total carbonyl content than those of the controls or moderate patients (fig. 1b).

\section{Carbonylated proteins}

The identification of the different carbonylated proteins revealed that muscle creatine kinase, muscle carbonic anhydrase III, $\alpha-1$ sarcomeric actin and MyHC were specifically oxidised in the diaphragms of both groups of patients and control subjects (table 2; fig. 1c and d; fig. 2). Carbonyl group
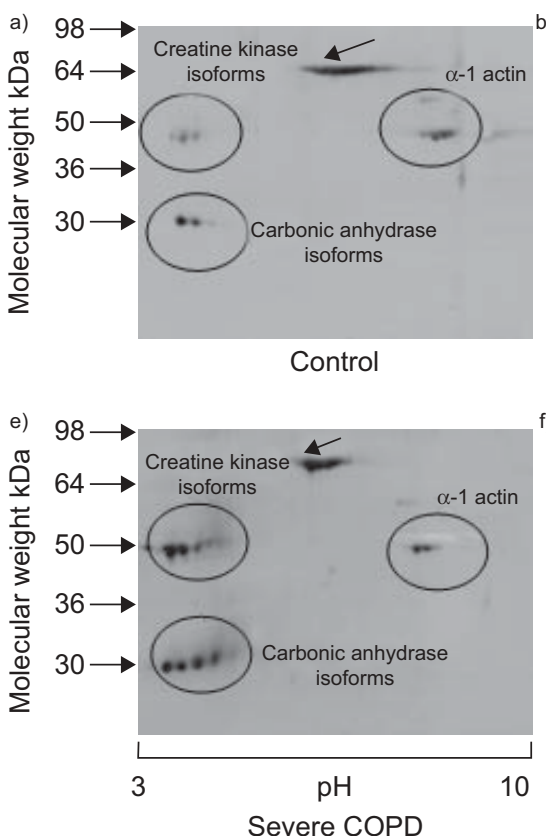

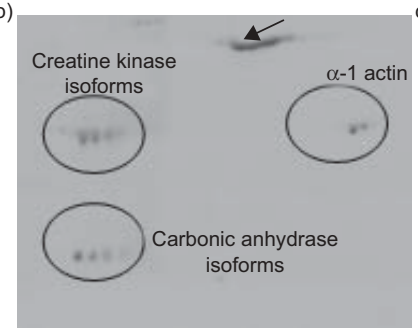

Control

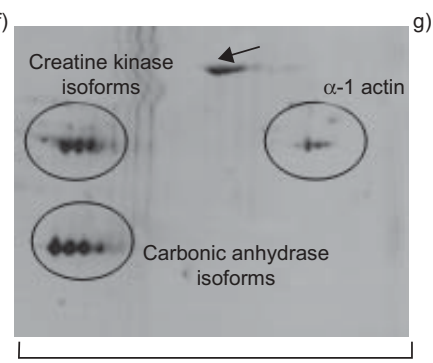

3

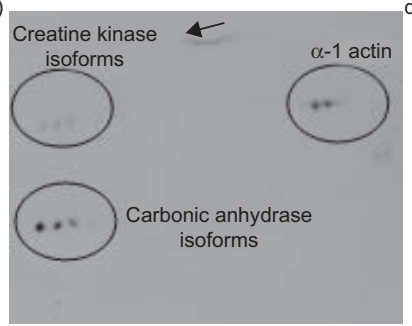

Moderate COPD

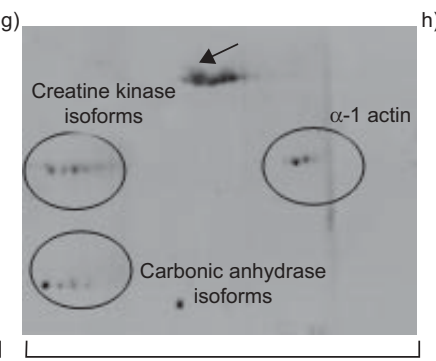

$\mathrm{pH}$

Severe COPD

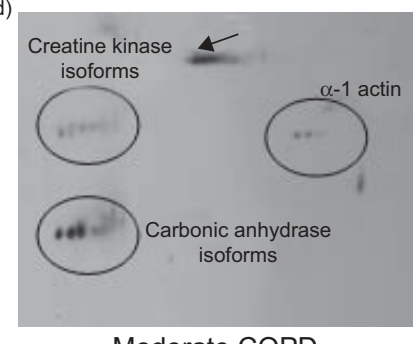

Moderate COPD

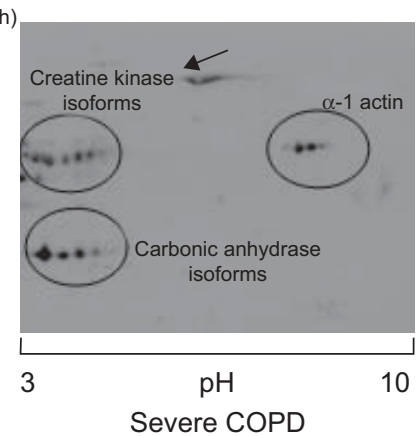

FIGURE 2. Representative two-dimensional immunoblots corresponding to the detection of carbonylated proteins in crude muscle homogenates of diaphragm muscles of two control subjects ( $\mathrm{a}$ and $\mathrm{b}$ ) and two patients with moderate chronic obstructive pulmonary disease (COPD; $\mathrm{c}$ and d) and four patients with severe COPD (e-h). Muscle creatine kinase, carbonic anhydrase III and $\alpha$-actin were consistently oxidised in the diaphragms of both patients and controls. Albumin was also oxidised in both patients and controls (arrow). 
a)
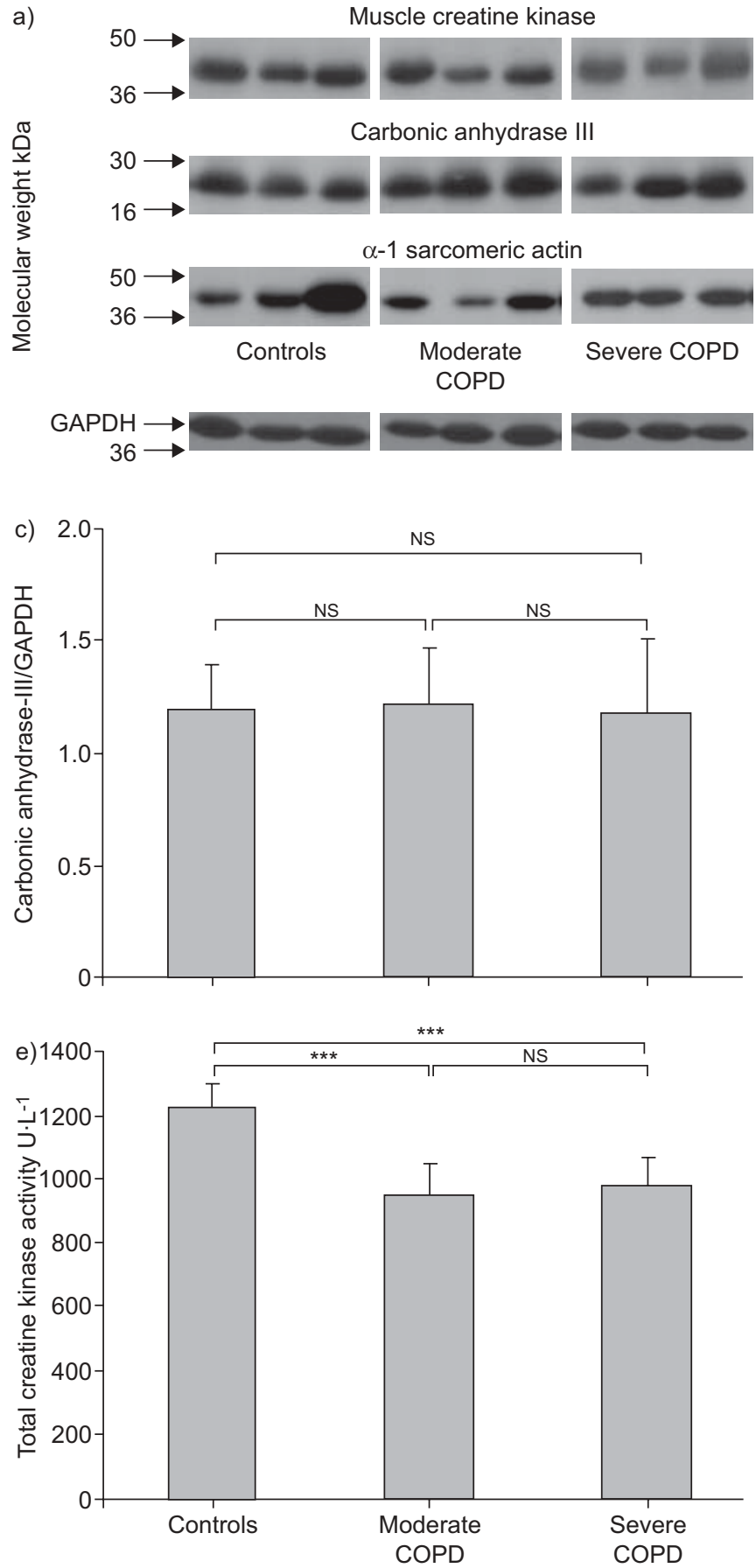

formation in $\mathrm{MyHC}$ protein was significantly greater in the diaphragms of the severe COPD patients compared with either moderate COPD or control subjects (fig. 1c and d). Indeed, there was a five-fold increase in MyHC carbonylation in the diaphragms of the patients with severe disease compared with the controls (fig. 1c and d). Albumin ( 58-67 kDa) was also consistently oxidised in all the diaphragm specimens (fig. 2).

\section{Expression and activity of identified carbonylated proteins}

Muscle creatine kinase content was significantly lower in the diaphragms of severe COPD patients compared with either
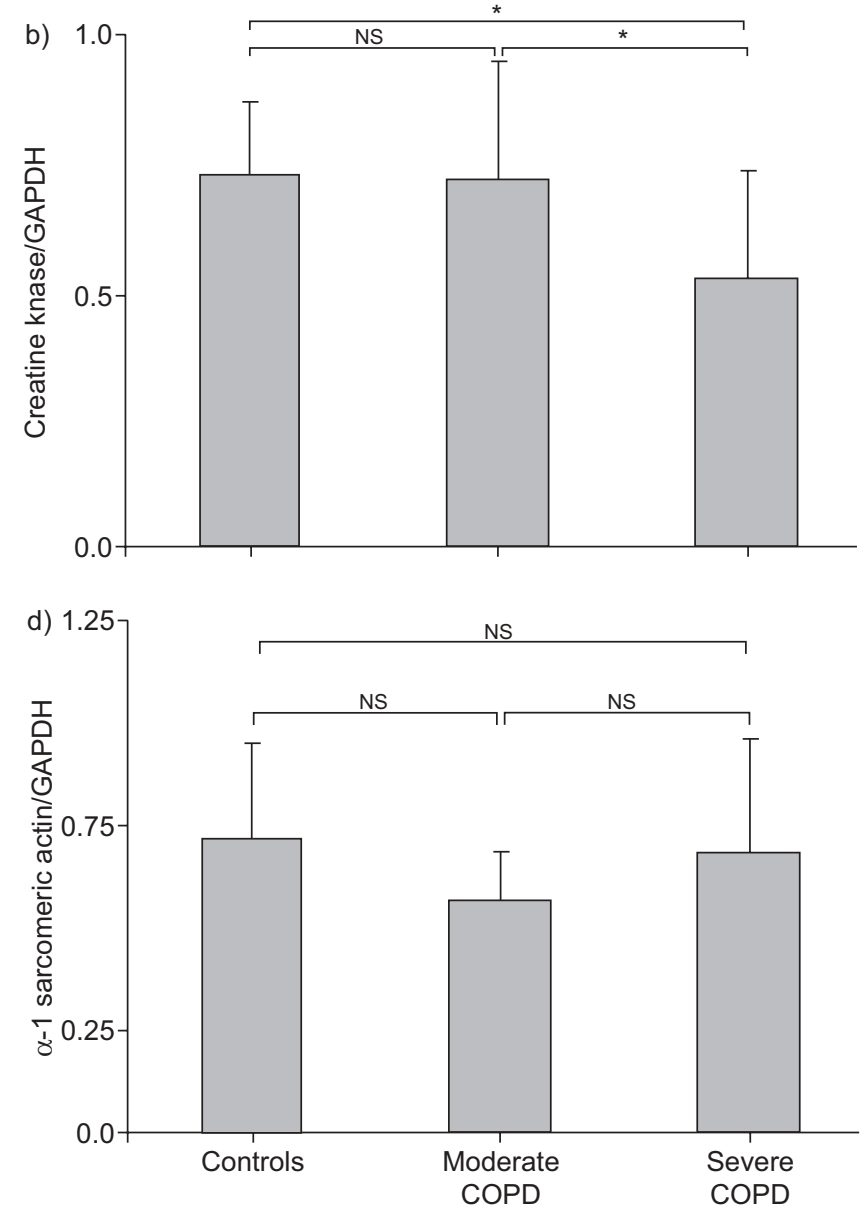

FIGURE 3. a) Representative examples of protein expression of muscle creatine kinase, carbonic anhydrase III, and $\alpha-1$ sarcomeric actin in the diaphragms of control subjects $(n=10)$ and both moderate $(n=8)$ and severe $(n=16)$ chronic obstructive pulmonary disease (COPD) patients. Monoclonal anti-GAPDH horseradish peroxidase conjugated antibody was used to control equal loading among various lanes. Mean $\pm S D$ values of b) creatine kinase content, c) carbonic anhydrase III content, d) $\alpha-1$ sarcomeric actin protein content and e) creatine kinase activity in diaphragm muscles of control subjects $(n=10)$ and both moderate $(n=8)$ and severe $(n=16)$ COPD patients. Note that creatine kinase content was significantly reduced in the diaphragms of the severe COPD patients compared with protein levels in the moderate patients and controls. No significant differences were found in carbonic anhydrase III or $\alpha-1$ sarcomeric actin among the three study groups. The enzyme activity of creatine kinase was significantly reduced in the diaphragms of the COPD patients, both moderate and severe, compared with activity levels in the control subjects. ${ }^{*}: p \leqslant 0.05 ; * * *: p \leqslant 0.001$. NS: nonsignificant.

moderate COPD patients or control subjects (fig. 3a and b). Protein levels of carbonic anhydrase III and actin were not significantly different between COPD patients and controls (fig. 3a, c and d). Moreover, creatine kinase activity was significantly reduced in the diaphragms of both severe and moderate COPD patients compared with controls (fig. 3e). Importantly, MyHC content was significantly reduced in the diaphragms of the severe COPD patients compared with either moderate COPD or control subjects (fig. 4). In fact, in the severe patients, diaphragm $\mathrm{MyHC}$ levels were $32 \%$ of those detected in the controls. 


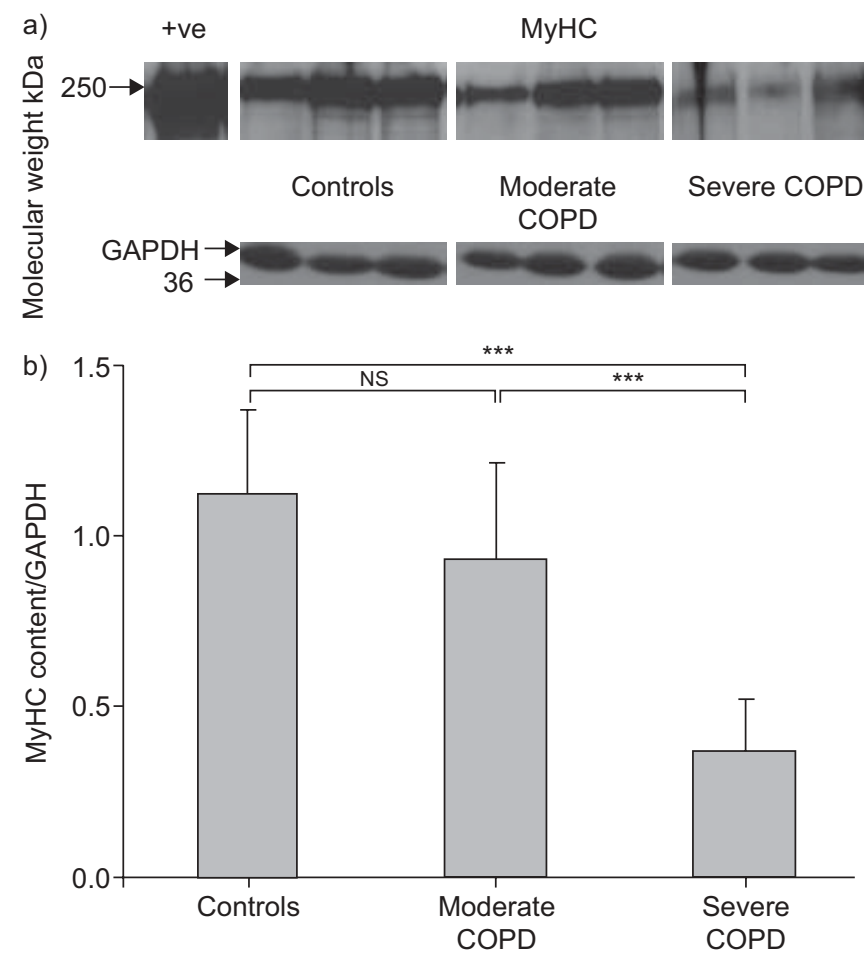

FIGURE 4. a) Representative examples of myosin heavy chain (MyHC) protein content in the diaphragms of control subjects $(n=9)$ and both moderate $(n=8)$ and severe $(n=9)$ chronic obstructive pulmonary disease (COPD) patients. Monoclonal anti-GAPDH horseradish peroxidase conjugated antibody was used to control equal loading among various lanes. c) MyHC protein content was lower in the diaphragms of the severe COPD patients compared with either controls or moderate patients. $\mathrm{MyHC}$ content did not differ between moderate patients and the controls. ${ }^{* *}: p \leqslant 0.001$. NS: nonsignificant.

\section{Protein tyrosine nitration}

Several nitrated proteins were identified with apparent masses ranging from 64 to $30 \mathrm{kDa}$ (fig. 5a). Levels of diaphragm protein nitration did not differ among the study groups (fig. 5b).

\section{Diaphragm superoxide anion production}

Diaphragm levels of superoxide anion were significantly higher in the mitochondrial fraction of severe COPD patients compared with those in the controls (fig. 6a). Likewise, muscle superoxide anion levels were greater in the membrane fraction of severe COPD patients compared with either moderate COPD patients or controls (fig. 6b). However, diaphragm superoxide anion production from the cytosolic fraction did not significantly differ between COPD patients and control subjects (fig. 6c).

Interestingly, the percentage of superoxide anion inhibition elicited by rotenone (respiratory chain inhibitor) was significantly greater in the diaphragms of both severe and moderate COPD patients compared with controls (fig. 7a). Conversely, the percentage of superoxide anion inhibition elicited by the inhibitors apocynin (specific nicotinamide adenine dinucleotide phosphate hydrogen (NADPH) oxidase inhibitor) and oxypurinol (inhibitor of xanthine oxidase),

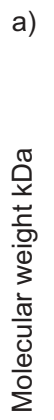

a)
$\frac{\pi}{2}$
$\frac{2}{1}$
$\frac{0}{0}$
$\frac{0}{0}$
$\frac{1}{5}$
$\frac{0}{0}$
$\frac{0}{0}$
$\frac{0}{2}$
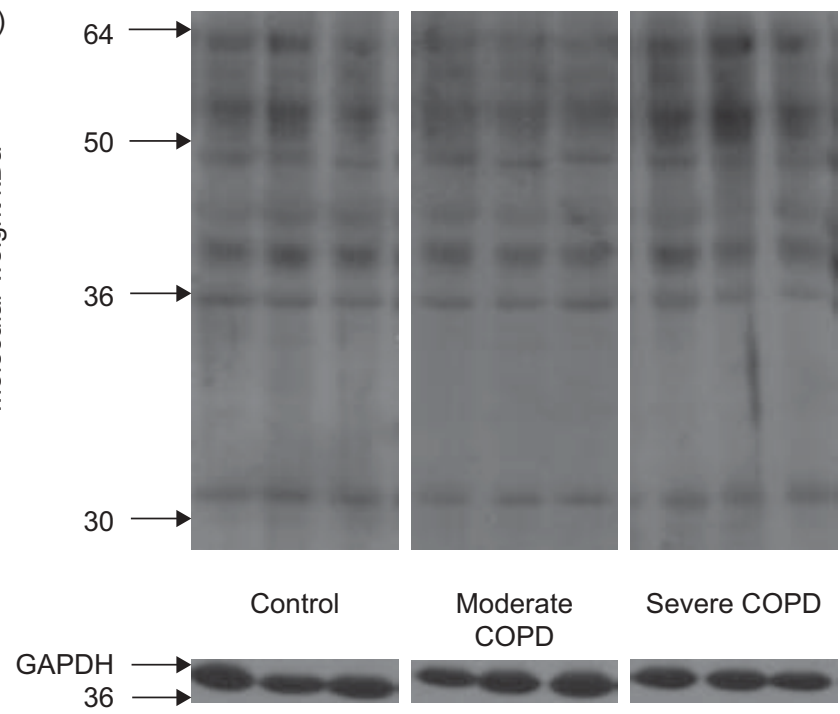

Moderate COPD

Severe COPD
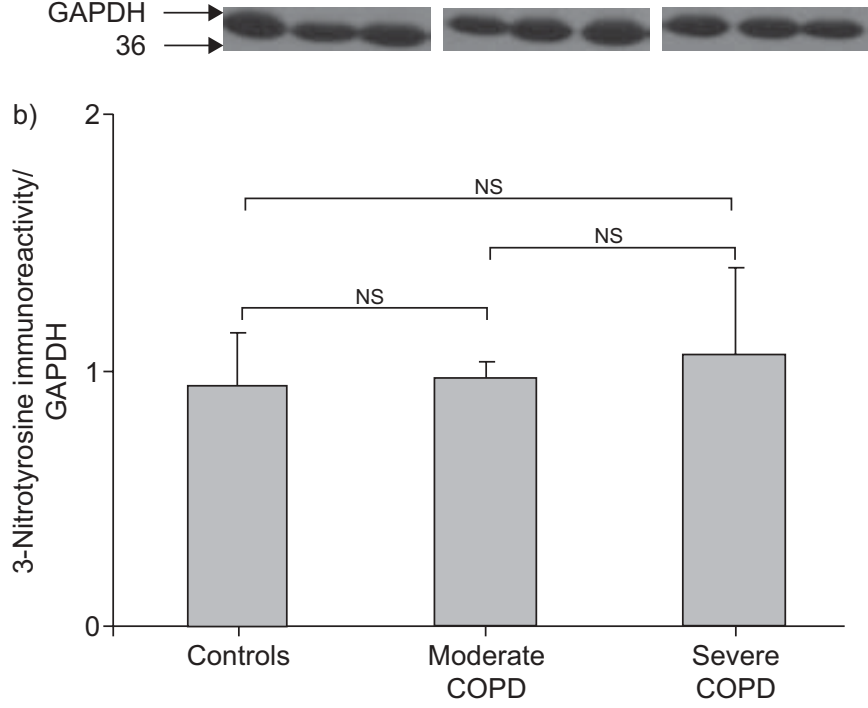

FIGURE 5. a) Representative examples of protein tyrosine nitration in diaphragms of control subjects $(n=10)$ and both moderate $(n=8)$ and severe $(n=16)$ chronic obstructive pulmonary disease (COPD) patients. Several nitrated protein bands were detected. Monoclonal anti-GAPDH horseradish peroxidase conjugated antibody was used to control equal loading among various lanes. b) Levels of diaphragm protein tyrosine nitration did not differ among the study groups. NS: nonsignificant.

respectively, did not differ between COPD patients and controls (fig. $7 \mathrm{~b}$ and c).

\section{DISCUSSION}

The novel findings in this study are that in the diaphragms of severe COPD patients compared with control subjects are the following: 1) total muscle protein carbonylation rather than protein nitration was increased; 2) muscle creatine kinase, carbonic anhydrase III, actin and myosin were oxidised; 3 ) carbonylation levels in MyHC were five-fold greater; 4) creatine kinase content and activity were reduced and MyHC levels were $32 \%$ of those in the controls; 5) superoxide anion levels were increased in the mitochondria and membrane compartments; and 6) the percentage of superoxide anion inhibition achieved by the mitochondrial inhibitor rotenone was significantly greater. 

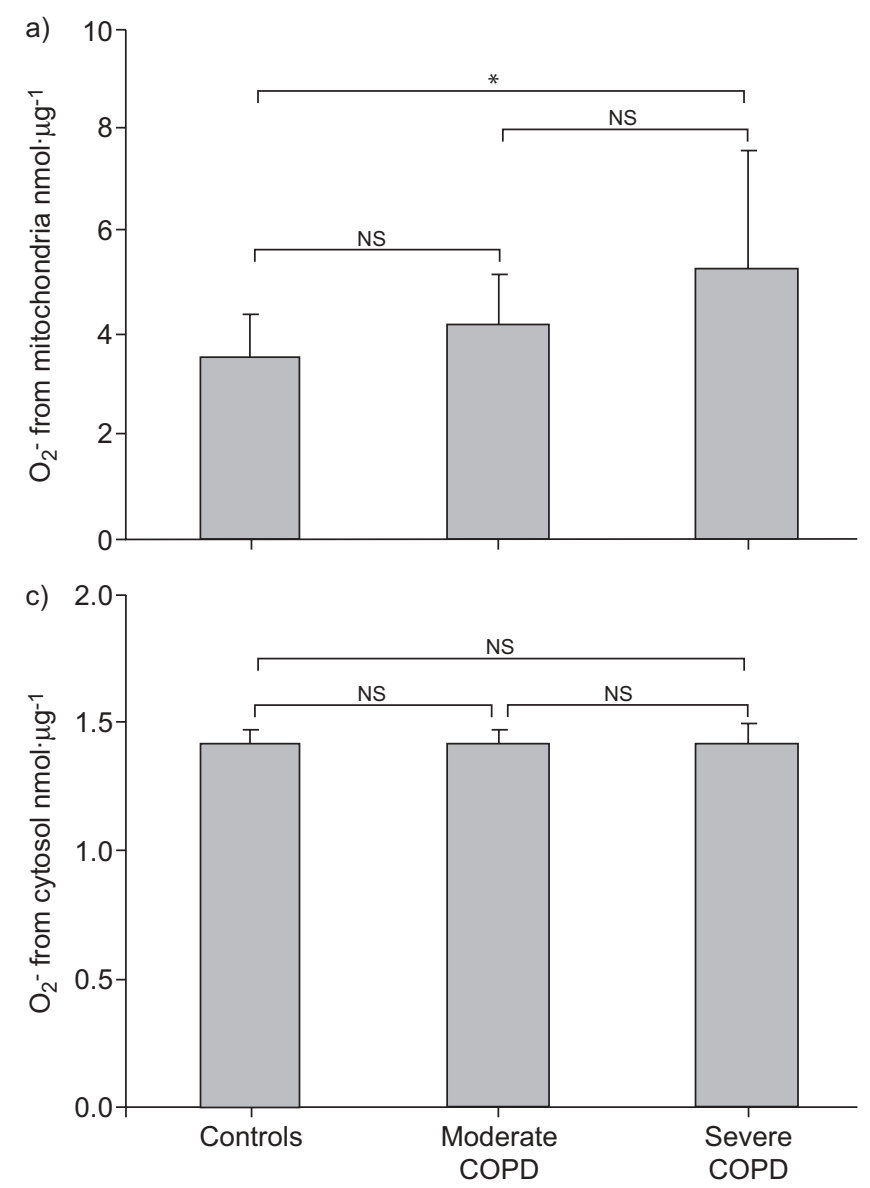

\section{Respiratory muscle dysfunction and structure}

In the current investigation, the severe COPD patients exhibited a moderate reduction in respiratory muscle strength, as measured by Pdi,max and PI,max. Although this level of respiratory muscle dysfunction may not be clinically relevant at rest, it could have significant clinical implications under certain circumstances, such as during an exacerbation or physical exercise. Importantly, our observations were consistent with previous reports, where in vivo respiratory muscle and diaphragm strength were also shown to be compromised $[14,21,22]$. In keeping with this, it has recently been proposed [23] that in severe COPD, several molecular mechanisms contribute to the aetiology of this respiratory muscle dysfunction, such as myosin loss [10, 11], sarcomeric injury [24], oxidative stress [14] and alterations in cross-bridge cycling kinetics [8], resulting in reduced diaphragm isometric force. This raises serious questions concerning whether the adaptive mechanisms encountered in the diaphragms of severe COPD patients [4-8] are sufficient to make this muscle more efficient, since impaired contractile properties of the diaphragm muscle fibres have been clearly demonstrated [9-11].

In the present study, the diaphragm fibre type shift towards a fatigue-resistant phenotype exhibited by the severe COPD patients is in agreement with previous investigations [4, 14]. Moreover, we provide evidence of the proportions of hybrid fibres in the human diaphragm. The significant increase in hybrid fibre proportions observed in the diaphragms of the

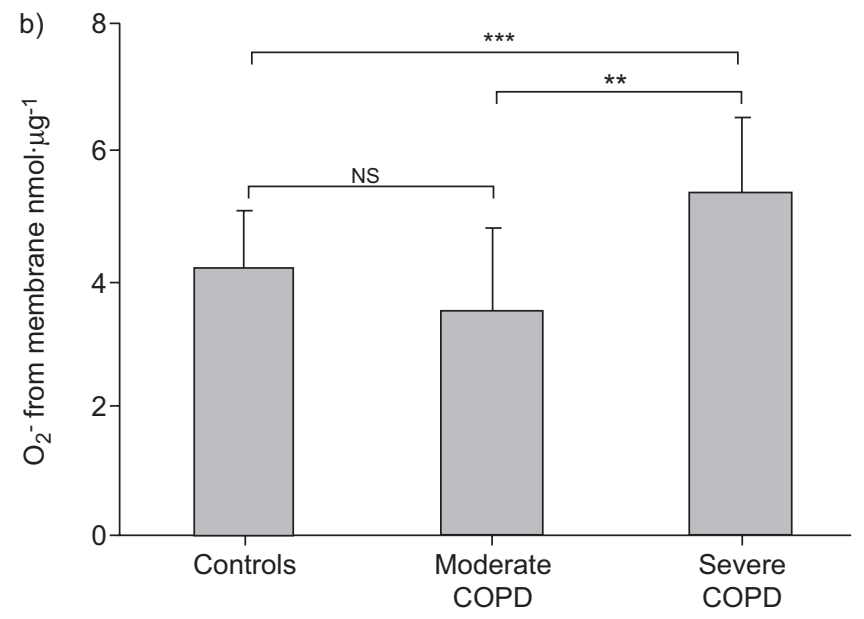

FIGURE 6. Mean $\pm S D$ values of superoxide anion $\left(\mathrm{O}_{2}{ }^{-}\right)$production in the a) mitochondrial, b) membrane and c) cytosolic compartments of control subjects $(n=10)$, moderate $(n=8)$ and severe $(n=16)$ chronic obstructive pulmonary disease (COPD) patients. The production of $\mathrm{O}_{2}^{-}$was significantly increased in the diaphragm mitochondrial fraction (a) of severe COPD patients compared with control subjects but not with moderate COPD patients. The production of $\mathrm{O}_{2}{ }^{-}$was also significantly increased in the diaphragm membrane fraction (b) of severe COPD patients compared with either moderate COPD or control subjects. No significant differences were observed in the production of diaphragm cytosolic $\mathrm{O}_{2}$ between COPD patients and controls (c). ${ }^{*}: p \leqslant 0.05 ;{ }^{*}: p \leqslant 0.01 ; * * *: p \leqslant 0.001$ NS: nonsignificant.

severe patients is likely to be another adaptive mechanism to their respiratory overloads. Interestingly, the size of the diaphragm fibres did not differ between patients and controls. In fact, discrepancies exist in the literature regarding diaphragm fibre cross-sectional areas in COPD patients. While in several studies the size of the diaphragm fibres was reduced in these patients $[4,6,25]$, no significant differences were found between patients and controls in other investigations $[5,24,26]$. The number of patients examined, which was greater in the latter studies and in the current study, the methodologies employed in each study, such as the use of frozen or paraffin-embedded tissues, or the identification of the muscle fibre types using histochemical or immunohistochemical procedures, and the absence of nutritional abnormalities in our patients, which have been shown to influence diaphragm fibre sizes [25, 27], could account for such discrepancies.

\section{Diaphragm carbonylated proteins}

Over the past decade, a growing body of evidence has shown that oxidative stress is one of the mechanisms clearly involved in the skeletal muscle dysfunction of COPD patients [12-14]. Carbonyl formation is an important detectable marker of protein oxidation in tissues. The present study is the first to report that carbonylation of diaphragm proteins in severe COPD and in control subjects includes proteins involved in key muscle cellular processes, such as adenosine triphosphate metabolism (creatine kinase), hydration of carbon dioxide 

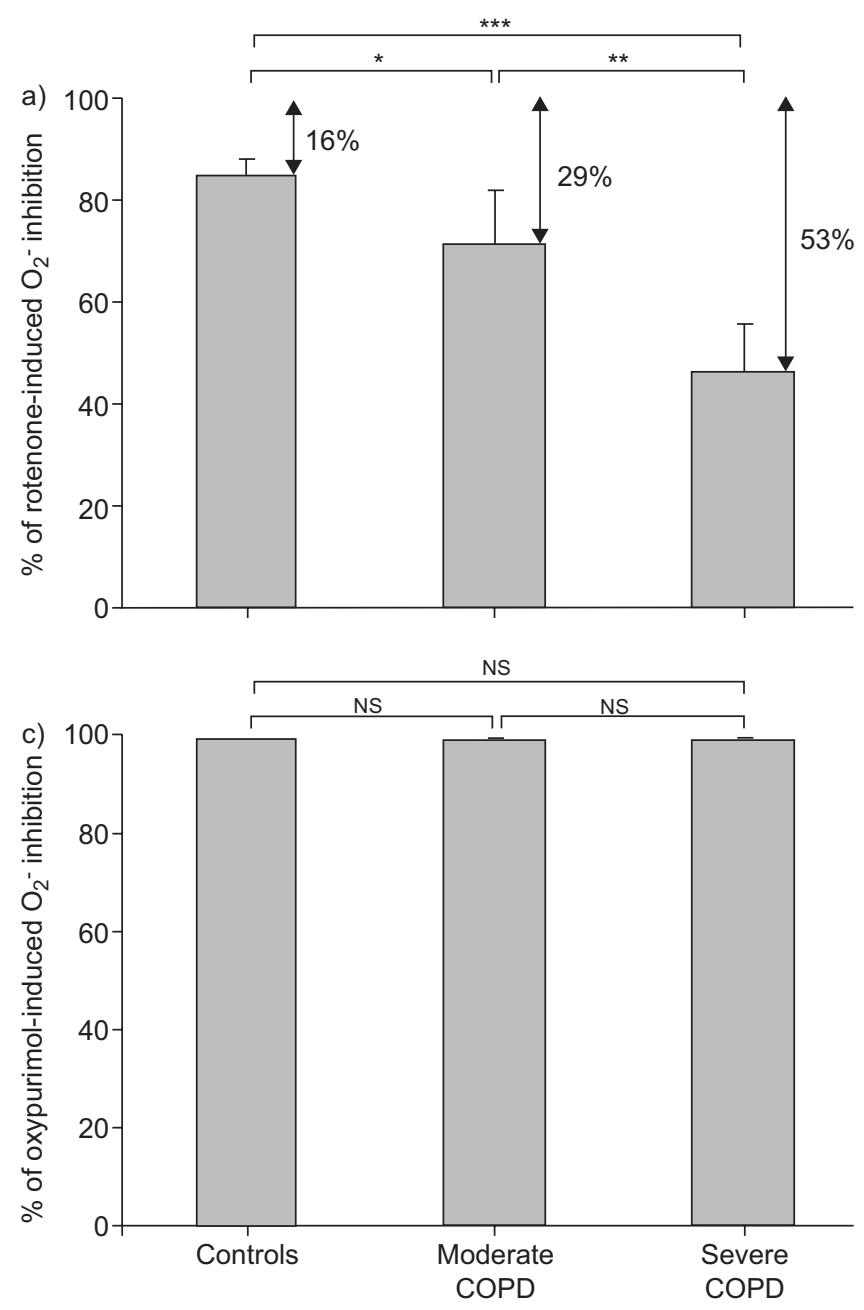

(carbonic anhydrase III) and contractile function (actin and $\mathrm{MyHC})$.

Diaphragm carbonic anhydrase III content was not different in COPD patients to that in controls. This is in keeping with a previous study [13], in which quadricep carbonic anhydrase levels did not differ between COPD patients and controls. Creatine kinase activity, however, was reduced in the diaphragms of both moderate and severe COPD patients. Indeed, this is consistent with previous reports, in which creatine kinase was shown to be a major target for the in vitro and in vivo exposure of ROS, resulting in enzyme inactivation $[28,29]$. In our study, although patients with moderate COPD did not exhibit a significant reduction in diaphragm creatine kinase content, it could be hypothesised that moderate levels of ROS in their muscles may have already influenced enzyme activity, since their levels were indeed similar to those of the severe patients. Moreover, it has been well established that slow-twitch oxidative fibres exhibit the lowest creatine kinase activity in skeletal muscles [30]. On this basis, it is possible to conclude that the decreased creatine kinase activity in the diaphragms of our severe COPD patients could be partly due to their fibre-type shift towards a more fatigue-resistant phenotype. Eventually, although in the current investigation it is not possible to define the precise contribution of reduced

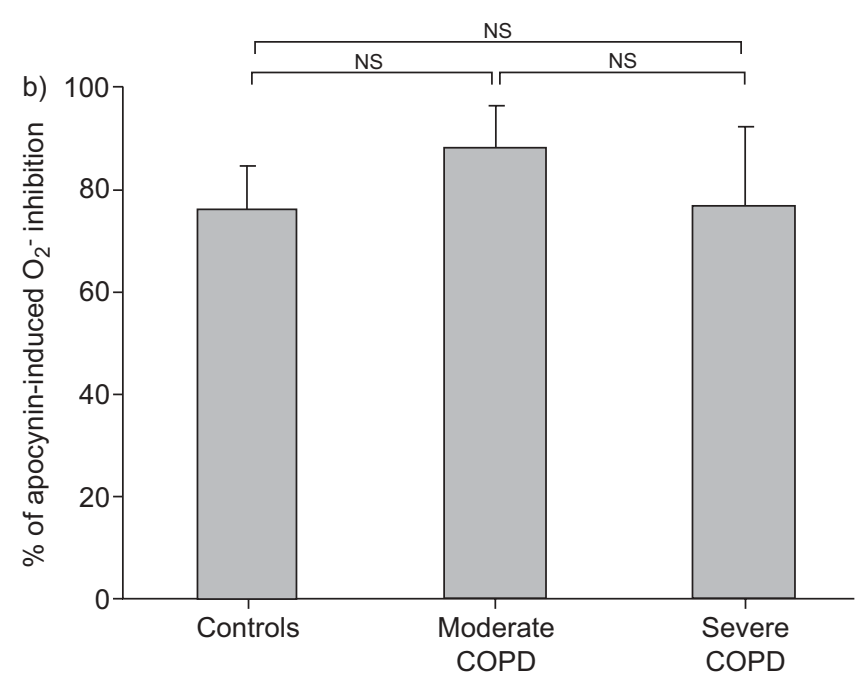

FIGURE 7. Mean \pm SD values of the percentage of superoxide anion $\left(\mathrm{O}_{2}{ }^{-}\right)$ inhibition induced by selective enzyme inhibitors such as a) rotenone, b) apocynin and c) oxypurinol. The percentage of $\mathrm{O}_{2}^{-}$inhibited by the mitochondrial respiratory chain inhibitor rotenone (a) was significantly greater in the diaphragms of the severe ( $n=16 ; 53 \%$ ) chronic obstructive pulmonary disease (COPD) patients compared with either controls $(n=10 ; 16 \%)$ or moderate $(n=8 ; 29 \%)$ COPD patients Interestingly, the percentage of $\mathrm{O}_{2}^{-}$inhibited by rotenone was also significantly greater in the diaphragms of the moderate patients compared with the controls. The percentage of $\mathrm{O}_{2}{ }^{-}$inhibited by the NADPH oxidase inhibitor apocynin (b) did not significantly differ between COPD patients and controls. Likewise, the percentage of $\mathrm{O}_{2}{ }^{-}$inhibited by the xanthine oxidase inhibitor oxypurinol (c) was not significantly different in the diaphragms of COPD patients compared with controls. *: $p \leqslant 0.05$; $* *: p \leqslant 0.01 ; * * *: p \leqslant 0.001$. Ns: nonsignificant.

creatine kinase activity to respiratory muscle dysfunction in severe COPD patients, it is likely to play a role, since absence of creatine kinase activity in mice led to profound reductions in exercise performance [31] and to myocardial dysfunction [32].

In contrast to the current findings, in a previous study [7] total creatine kinase activity levels in the diaphragms of severe COPD patients were shown not to differ from those in the control muscles. Discrepancies between the two studies could be explained by the different methodologies employed to determine creatine kinase activity and by the fact that measurements were only conducted in saponin-skinned fibres in the previous study [7]. In line with the current findings, the quadriceps of severe COPD patients also exhibited a significant reduction in creatine kinase content and activity both at rest [13] and after exercise training [33]. However, creatine kinase activity in the deltoid [34] and external intercostal muscles [35] did not differ between severe to moderate COPD patients and control subjects.

Several studies have already demonstrated oxidation of the cytoskeletal protein actin in different models [36, 37]. The complex modifications induced by ROS on muscle actin are characterised by severe disruption of the actin filaments, hampering their interaction with the myosin protein [38], thus 
suggesting that oxidation of contractile actin may contribute to muscle dysfunction in these models $[36,38]$. In our study, although muscle actin levels did not differ between COPD patients and controls, disruption of the contractile actin in the diaphragms of the severe patients cannot be ruled out.

Importantly, in line with the observations of OTTENHEIJM and co-workers $[10,11]$, the diaphragm of our severe COPD patients exhibited significantly lower levels (32\%) of $\mathrm{MyHC}$ protein than the controls. The novel finding of the current study is that in the diaphragms of the severe patients, carbonylation levels of $\mathrm{MyHC}$ protein were five times greater than in the control muscles. Although increased $\mathrm{MyHC}$ carbonylation and reduced MyHC content in the diaphragms of our severe patients cannot be causally related, it is likely that the greater oxidative damage exhibited by $\mathrm{MyHC}$ protein in these muscles may have contributed to its higher protein breakdown [10, 11]. In fact, activity of the ubiquitinproteasome pathway was shown to be increased in patients with mild to moderate COPD [11] and inflammatory cytokines and ROS are well-known triggers of muscle proteolysis [16]. Taken together, these findings suggest that, in the diaphragms of severe COPD patients, highly carbonylated MyHC protein is prone to further degradation by the ubiquitin-proteasome pathway. Clearly, a future study should be designed in order to explore the exact contribution of oxidants to increased muscle protein breakdown and myosin loss in the diaphragms of severe COPD patients.

\section{Diaphragm superoxide anion formation}

The current investigation is the first to report in vitro measurements of the superoxide anion being produced within distinct muscle fractions in the human diaphragm. Interestingly, superoxide anions are the initial substrates of a series of reactions resulting in the formation of more powerful ROS in tissues. In the present study, in vitro generation of the superoxide anion by the mitochondrial and membrane compartments was significantly increased in the diaphragms of severe COPD patients compared with controls. Another important finding in this study is that rotenone, a selective mitochondrial respiratory chain inhibitor, elicited significantly greater inhibitory effects of ROS generation in the diaphragms of the patients. Importantly, in exercising muscles, oxygen utilisation increases several fold, eventually resulting in leakage of superoxide anions from the mitochondria to other muscle structures. In patients with severe COPD, the main inspiratory muscle, the diaphragm, must incessantly generate muscle contractions of relatively high intensity in order to overcome the increased inspiratory loads imposed by the mechanical alterations of their thoracic cage. This may result in the continuous generation of abnormally high levels of superoxide anion within the diaphragm mitochondria, which would not possibly revert to "control" levels in these patients.

In view of the significant increase in superoxide anion generation in the membrane compartment of diaphragms in the severe COPD patients, it may be suggested that muscle NADPH oxidase enzyme complex was a likely contributor to superoxide anion generation in these muscles, as has previously been shown in septic muscles [20]. In contrast to the mitochondrial and membrane compartments, the amount of superoxide anion produced in the cytosolic fraction was relatively low in the diaphragms of both patients and controls. Furthermore, oxypurinol had no major effects on ROS generation within the diaphragm cytosolic compartment in any of the study groups, suggesting that xanthine oxidase is probably not involved in the generation of superoxide anion in the human diaphragm.

\section{Study limitations}

Although lung volume reduction surgery also makes it possible to obtain diaphragm specimens, only very severe COPD patients undergo this type of surgery. Therefore, diagnostic-therapeutic thoracotomy is the only approach available for studying mild to moderate COPD and normal lung function subjects. Accordingly, the population of our study shares a common morbidity; the presence of a localised lung neoplasm. Nevertheless, we do not believe that this condition has made any significant contribution to the development of oxidative stress in these diaphragms, since extremely restrictive criteria were employed to select our population. Another limitation encountered in our study is directly related to the methodologies involved in the in vitro measurements of muscle superoxide anion, which have been challenged on the basis of the potential production of superoxide anion by the redox recycling of lucigenin. However, as previously demonstrated [20], we do not believe that lucigenin redox recycling has made any substantial contribution to the superoxide anion concentrations detected in our study.

In the present study, both patients and controls were recruited over a period of several years. In order to avoid unnecessary methodological concerns due to analyses conducted sequentially rather than simultaneously, all muscle specimens were always stored frozen at $-80^{\circ} \mathrm{C}$ until further use in the laboratory. Although we acknowledge that freezing and fractionation of the muscle samples might have somehow influenced the superoxide anion measurements, we do not believe that this has had any substantial effects in our study for the following reasons: 1) All superoxide anion analyses were conducted simultaneously for both patients and controls under identical laboratory conditions by the same investigator; 2) a fragment of the muscle specimens was always kept frozen in our $-80^{\circ} \mathrm{C}$ freezer until muscle fractions were obtained and superoxide anion experiments were conducted; and 3) fractionation of the muscle samples using specific buffers that do not destroy the different cell compartments was already demonstrated to yield reliable lucigenin-derived chemiluminescence signals [20]. Further information can be found in the online supplementary material.

\section{Conclusions}

Our study is the first to provide evidence that in severe COPD patients, oxidation of diaphragm proteins involved in energy production and contractile performance is likely to partially contribute to the documented respiratory muscle dysfunction. Furthermore, generation of the superoxide anion was increased in the diaphragms of these patients.

\section{ACKNOWLEDGEMENTS}

The authors are grateful to M.L. Blanco (IMIM-Hospital del Mar), M.J. Masdeu (Hospital Parc Taulí, Sabadell), J.A. Barberà (Hospital Clinic), and J. Martínez-Llorens (IMIM-Hospital del 
Mar) for their help with the patient recruitment, C. Chiva and M. Rodríguez from the Protemics Laboratory (Universtat Pompeu Fabra) for their help with the protein identification, to F. Sánchez and S. Mas (both IMIM-Hospital del Mar; all Barcelona, Spain) for their technical assistance in the laboratory, and to R. Marshall for help with editing.

\section{REFERENCES}

1 Ferrer M, Alonso J, Morera J, et al. Chronic obstructive pulmonary disease stage and health-related quality of life. Ann Intern Med 1997; 127: 1072-1079.

2 Feenstra TL, van Genugten ML, Hoogenveen RT, et al. The impact of aging and smoking on the future burden of chronic obstructive pulmonary disease: a model analysis in the Netherlands. Am J Respir Crit Care Med 2001; 164: 590-596.

3 Halbert RJ, Natoli JL, Gano A, et al. Global burden of COPD: systematic review and meta-analysis. Eur Respir J 2006; 28: 523-532.

4 Levine S, Kaiser L, Leferovich J, et al. Cellular adaptations in the diaphragm in chronic obstructive pulmonary disease. N Engl J Med 1997; 337: 1799-1806.

5 Orozco-Levi M, Gea J, Lloreta JL, et al. Subcellular adaptation of the human diaphragm in chronic obstructive pulmonary disease. Eur Respir J 1999; 13: 371-378.

6 Levine S, Gregory C, Nguyen T, et al. Bioenergetic adaptation of individual human diaphragmatic myofibers to severe COPD. J Appl Physiol 2002; 92: 1205-1213.

7 Ribera F, N' Guessan B, Zoll J, et al. Mitochondrial electron transport chain function is enhanced in inspiratory muscles of patients with chronic obstructive pulmonary disease. Am J Respir Crit Care Med 2003; 167: 873-879.

8 Stubbings AK, Moore AJ, Dusmet M, et al. Physiological properties of human diaphragm muscle fibres and the effect of chronic obstructive pulmonary disease. J Physiol 2008; 586: 2637-2650.

9 Levine S, Nguyen T, Kaiser LR, et al. Human diaphragm remodeling associated with chronic obstructive pulmonary disease. Am J Respir Crit Care Med 2003; 168: 706-713.

10 Ottenheijm CA, Heunks LM, Sieck GC, et al. Diaphragm dysfunction in chronic obstructive pulmonary disease. Am J Respir Crit Care Med 2005; 172: 200-205.

11 Ottenheijm CA, Heunks LM, Li YP, et al. Activation of the ubiquitin-proteasome pathway in the diaphragm in chronic obstructive pulmonary disease. Am J Respir Crit Care Med 2006; 174: 997-1002.

12 Koechlin C, Couillard A, Simar D, et al. Does oxidative stress alter quadriceps endurance in chronic obstructive pulmonary disease? Am J Respir Crit Care Med 2004; 169: 1022-1027.

13 Barreiro E, Gea J, Matar G, et al. Expression and carbonylation of creatine kinase in the quadriceps femoris muscles of patients with chronic obstructive pulmonary disease. Am J Respir Cell Mol Biol 2005; 33: 636-642.

14 Barreiro E, de la Puente B, Minguella J, et al. Oxidative stress and respiratory muscle dysfunction in severe chronic obstructive pulmonary disease. Am J Respir Crit Care Med 2005; 171: 1116-1124.

15 Souza JM, Choi I, Chen Q, et al. Proteolytic degradation of tyrosine nitrated proteins. Arch Biochem Biophys 2000; 380: 360-366.
16 Powers SK, Kavazis AN, McClung JM. Oxidative stress and disuse muscle atrophy. J Appl Physiol 2007; 102: 2389-2397.

17 Rabe KF, Hurd S, Anzueto A, et al. Global strategy for the diagnosis, management, and prevention of chronic obstructive pulmonary disease: GOLD executive summary. Am J Respir Crit Care Med 2007; 176: 532-555.

18 Barreiro E, Gea J, Di Falco M, et al. Protein carbonyl formation in the diaphragm. Am J Respir Cell Mol Biol 2005; 32: 9-17.

19 Ohara Y, Peterson TE, Harrison DG. Hypercholesterolemia increases endothelial superoxide anion production. J Clin Invest 1993; 91: 2546-2551.

20 Javesghani D, Magder SA, Barreiro E, et al. Molecular characterization of a superoxide-generating $\mathrm{NAD}(\mathrm{P}) \mathrm{H}$ oxidase in the ventilatory muscles. Am J Respir Crit Care Med 2002; 165: 412-418.

21 Similowski T, Yan S, Gauthier AP, et al. Contractile properties of the human diaphragm during chronic hyperinflation. N Engl J Med 1991; 325: 917-923.

22 Polkey MI, Kyroussis D, Hamnegard CH, et al. Diaphragm strength in chronic obstructive pulmonary disease. Am J Respir Crit Care Med 1996; 154: 1310-1317.

23 Ottenheijm CA, Heunks LM, Dekhuijzen PN. Diaphragm muscle fiber dysfunction in chronic obstructive pulmonary disease: toward a pathophysiological concept. Am J Respir Crit Care Med 2007; 175: 1233-1240.

24 Orozco-Levi M, Lloreta J, Minguella J, et al. Injury of the human diaphragm associated with exertion and chronic obstructive pulmonary disease. Am J Respir Crit Care Med 2001; 164: 1734-1739.

25 Sánchez J, Medrano G, Debesse B, et al. Muscle fibre types in costal and crural diaphragm in normal men and in patients with moderate chronic respiratory disease. Bull Eur Physiopathol Respir 1985; 21: 351-356.

26 Doucet M, Debigaré R, Joanisse DR, et al. Adaptation of the diaphragm and the vastus lateralis in mild-to-moderate COPD. Eur Respir J 2004; 24: 971-979.

27 Kelsen SG, Ference M, Kapoor S. Effects of prolonged undernutrition on structure and function of the diaphragm. J Appl Physiol 1985; 58: 1354-1359.

28 Konorev EA, Hogg N, Kalyanaraman B. Rapid and irreversible inhibition of creatine kinase by peroxynitrite. FEBS Lett 1998; 427: 171-174.

29 Koufen P, Rück A, Brdiczka D, et al. Free radical-induced inactivation of creatine kinase: influence on the octameric and dimeric states of the mitochondrial enzyme (Mib-CK). Biochem J 1999; 344: 413-417.

30 Yamashita K, Yoshioka T. Profiles of creatine kinase isoenzyme compositions in single muscle fibres of different types. J Muscle Res Cell Motil 1991; 12: 37-44.

31 Momken I, Lechêne $\mathrm{P}$, Koulmann $\mathrm{N}$, et al. Impaired voluntary running capacity of creatine kinase-deficient mice. J Physiol 2005; 565: 951-964.

32 Spindler M, Engelhardt S, Niebler R, et al. Alterations in the myocardial creatine kinase system precede the development of contractile dysfunction in $\beta 1$-adrenergic receptor transgenic mice. J Mol Cell Cardiol 2003; 35: 389-397.

33 Puente-Maestu L, Tena T, Trascasa C, et al. Training improves muscle oxidative capacity and oxygenation 
recovery kinetics in patients with chronic obstructive pulmonary disease. Eur J Appl Physiol 2003; 88: 580-587.

34 Gea JG, Pasto M, Carmona MA, et al. Metabolic characteristics of the deltoid muscle in patients with chronic obstructive pulmonary disease. Eur Respir J 2001; 17: 939-945.

35 Pastó M, Gea J, Blanco M, et al. Metabolic activity of the external intercostal muscle of patients with COPD. Arch Bronconeumol 2001; 37: 108-114.
36 Powell SR, Gurzenda EM, Wahezi SE. Actin is oxidized during myocardial ischemia. Free Radic Biol Med 2001; 30: 1171-1176.

37 O'Reilly PJ, Hickman-Davis JM, Davis IC, et al. Hyperoxia impairs antibacterial function of macrophages through effects on actin. Am J Respir Cell Mol Biol 2003; 28: 443-450.

38 Kim E, Bobkova E, Hegyi G, et al. Actin cross-linking and inhibition of the actomyosin motor. Biochemistry 2002; 41: 86-93. 